\title{
DESIGN OF ELECTRON BEAM INJECTION SYSTEM FOR LASER ACCELERATION EXPERIMENTS AT JAERI-KANSAI
}

\author{
M. Kando ${ }^{*}$, H. Kotaki, H. Dewa, S. Kondo, and T. Hosokai \\ JAERI, Tokai, Ibaraki 319-1106, Japan \\ F. Sakai, J. Yang, and T. Hori,SHI, Tanashi, Tokyo 188-8585, Japan \\ K. Nakajima, KEK, Oho, Tsukuba, Ibaraki 305-0801, Japan
}

\begin{abstract}
We have designed an electron beam injection system for laser wakefield acceleration experiments which will be performed in a new facility of JAERI-Kansai in Kyoto. This system consists of an RF photocathode gun which can produce $5 \mathrm{ps} 1 \pi \mathrm{mm} \cdot \mathrm{mrad}$ electron single bunch and racetrack microtron (RTM). Here we have estimated an acceptance of laser wakefield in order to achieve more efficient acceleration, and have designed the high quality beam injector.
\end{abstract}

\section{INTRODUCTION}

Recent progress of high peak power laser technology enables us to study laser wakefield acceleration (LWFA) experimentally, which is a promising method for a high gradient compact accelerator. Several groups in the world have succeeded in acceleration of electrons by a laser wakefield so far[1]. However, the quality of the accelerated electrons such as energy spread, the number of accelerated particles, and so on, are not satisfied for practical applications as an accelerator. The major reason is because beam matching to the wakefield is not taken into account, or at least not sufficient, in the former experiments. In a typical LWFA experiment, a $100 \mathrm{fs}$ multi-tera watt laser pulse is focused into tens of microns. At a first look, it could be say that the LWFA requires an electron bunch to have the same order of both transverse and temporal sizes as those of the laser pulse.

There seems to be two approaches to accomplish perfect beam matching to the laser wakefield. One is a plasma cathode[2] which has a great potential in producing such high quality beam, because it produces electrons by a similar process of LWFA. However, this technique has not experimentally proved yet and must be tested separately. The other is to use a conventional accelerator for the injection. There exist some difficulties in producing ultra short bunches, but the recent progress of short bunch generation will help it[3].

In order to improve quality of beams accelerated by the wakefield we aim to develop a low emittance short bunch electron injector. For this purpose, a combination of the photocathode RF gun and the $150 \mathrm{MeV}$ race-track mi-

\footnotetext{
*Email:kando@apr.jaeri.go.jp
}

crotron (RTM) produces such a high quality beam with an appropriate budget. We have accomplished the photocathode RF gun capable of producing a $5 \mathrm{ps}$, less than $10 \pi$ $\mathrm{mm} \cdot \mathrm{mrad}$ beam with a charge of $1 \mathrm{nC}[4]$.

In this paper, we present a simple estimation of acceptance of the laser wakefield by a particle tracking code. Then we describe the injection system and improvements in order to make a low emittance beam in transverse direction.

\section{ACCEPTANCE OF LASER WAKEFIELD ACCELERATOR}

We have developed a particle tracking computer code named WAKE3D in order to examine acceptances of the laser wakefield accelerator. This code integrates three dimensional equation of motion where electric fields are given by a linear fluid theory. The integration length is 20 times of the vacuum Rayleigh length. No space-charge effect is included in the code.

Here, we only show typical results of the acceptance for a plasma density of $n_{e}=3.48 \times 10^{17} \mathrm{~cm}^{-3}$, a laser pulse length of $\tau_{L}=100 \mathrm{fs}$, a laser pulse energy of $E_{p}=500 \mathrm{~mJ}$, and a spot-size at focus of $\sigma_{r 0}=10 \mu \mathrm{m}$. Figure 1 shows the energy gain versus the initial phase. The initial electrons are injected to the wakefield on axis with energies of $1.3,15$, and $151 \mathrm{MeV}$. In case of the injected electrons are relativistic $(E>15 \mathrm{MeV})$, no bunching effect is seen in the figure. Our case is $151 \mathrm{MeV}$, thus, short bunch about 30 degrees ( $15 \mathrm{fs}$ ) is required for an energy gain of 40-60 $\mathrm{MeV}$. Transverse acceptance is shown in Fig. 2. Opposite to the longitudinal, transverse acceptance is wide enough for our system.

Generation of such tens of femtosecond electron pulse has not been achieved. However, we would obtain an improved energy spectrum in Fig. 3 if only the transverse beam matching is achieved. In this case, two side-peaks are seen, which are accelerated or decelerated by the wakefield, while the spectra of the former experiments obeyed power law as a function of an energy gain[1].

\section{ELECTRON INJECTION SYSTEM}

Figure 4 shows a schematic of the designed electron injection system. The system consists of the RF photocathode 


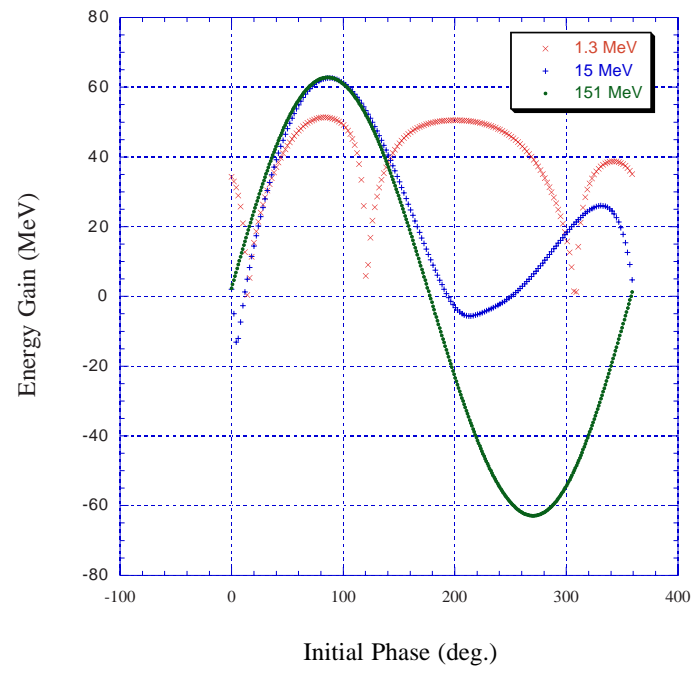

Figure 1: $\Delta E-\phi$ Phase space plot at the exit of the wakefield.

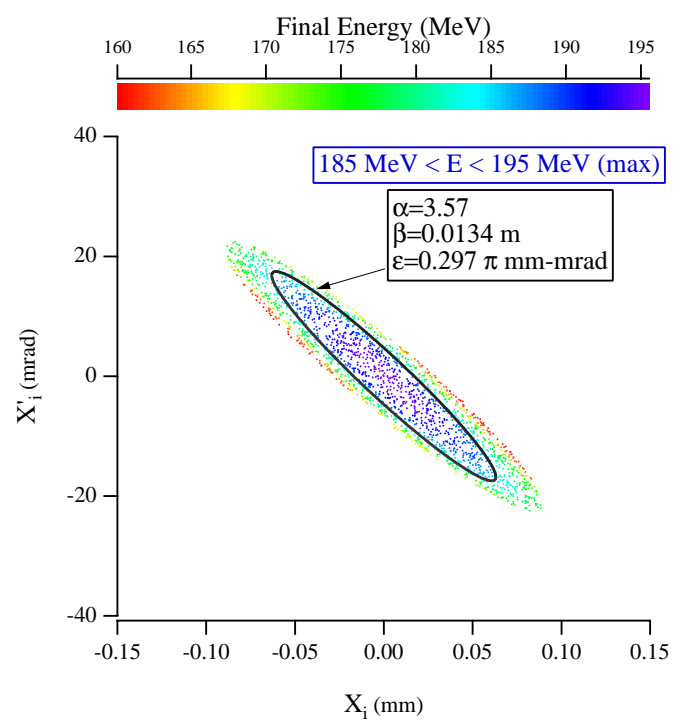

Figure 2: Transverse acceptance of the laser wakefield.

gun, the microtron accelerator and the beam extraction section.

\subsection{Photocathode RF Gun}

We adopted an S-band RF gun developed by the collaboration with BNL, KEK and Sumitomo Heavy Industries, Ltd (SHI). This cavity is based on the gun developed by the BNL / SLAC / UCLA collaboration [5] and is improved for high duty operation $(50 \mathrm{~Hz})$. The gun can produce $\sim 1 \pi$ $\mathrm{mm} \cdot \mathrm{mrad}$ electron pulses owing to an emittance compensation solenoid magnet. An UV light is introduced to the copper cathode with an incident angle of $68^{\circ}$.

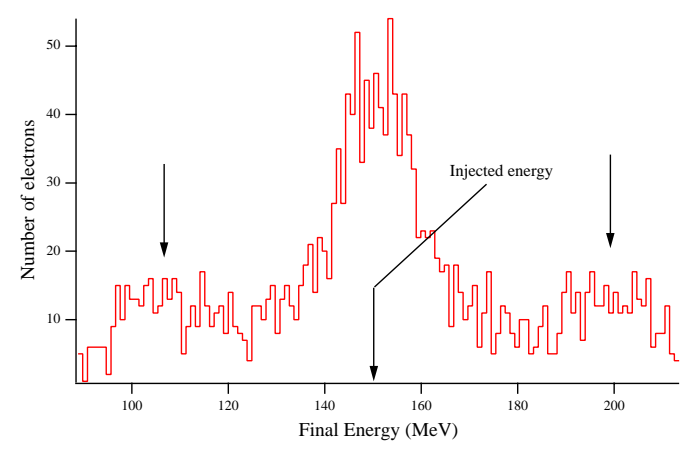

Figure 3: Energy spectrum of the beam matched only in transverse.

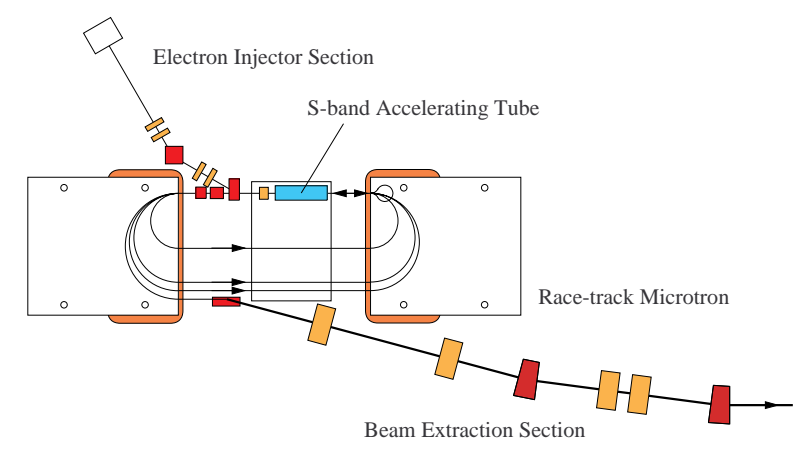

Figure 4: A schematic of the electron injection system.

\subsection{Photocathode Driving Laser}

We use a compact all solid-state Nd:YLF laser system (PULRISE II) developed by SHI as a driving laser for the RF gun. This system consists of three sections; passive mode-locked seed-laser with a repetition rate of 79.3 $\mathrm{MHz}$, laser diode (LD) pumped regenerative amplifier (regen) where a Nd:YLF crystal cut is used as a gain medium and frequency conversion sections.

Seed-laser pulses with a wavelength of $1053 \mathrm{~nm}$ are synchronized with an external reference signal $(79.3 \mathrm{MHz})$ by using a phase-locked loop. The regen section captures and amplifies a single seed-laser pulse increasing the pulse energy from the order of sub-nano Joule up to $2 \mathrm{~mJ}$ with a maximum repetition rate of $100 \mathrm{~Hz}$. The frequency conversion section generates a green light $(527 \mathrm{~nm})$ and an UV $(263 \mathrm{~nm})$. The output pulse energy of the UV is $200 \mu \mathrm{J}$ at maximum. The energy fluctuation of the UV is $0.5 \%$. The pulse width of the UV is approximately 6 ps FWHM.

\subsection{Test of Photocathode RF Gun}

We have performed tests of the photocathode RF gun and the driving laser which were installed at the Nuclear Engineering Research Laboratory, the University of Tokyo. The details of the results will be presented in forthcoming publication. As a preliminary result, we measured the electron pulse with a pulse length of 5 ps FWHM, an normalized 
emittance of $8 \pi \mathrm{mm} \cdot \mathrm{mrad}$, and an energy of $3.7 \mathrm{MeV}$.

\subsection{Microtron}

The race-track microtron (RTM) manufactured by SHI is used as a booster accelerator. The original RTM accelerates electrons emitted by a thermionic gun from $120 \mathrm{keV}$ to 150 $\mathrm{MeV}$ after 25 turns. We plan to replace the thermionic gun with the photocathode RF gun, thus some improvements should be required.

First, we estimated the acceptance of the RTM for 4.5 $\mathrm{MeV}$ injection using a code $M I C$ which was developed to design the original RTM[6]. After adjusting the position of the accelerating tube and injection timing, we found that a suitable electron beam is parallel, slightly converging, and $6 \mathrm{ps}$ in horizontal, vertical, and longitudinal direction, respectively (see Fig. 5).

The injection beamline to the RTM is designed to match the acceptance. We designed two bend achromat beamline by employing the transfer matrix, TRACE-3D, and PARMELA calculations. We obtained beam distributions shown in Fig. 6 by the MIC code after 25 turns in the RTM. Finally, we found that horizontal and vertical acceptances are 0.33 and $0.13 \pi \mathrm{mm}$-mrad, respectively.

Thus, transverse beam matching

will be achieved by an appropriate focusing section, which is under design. The pulse length is 3 ps FWHM for the present design, thus, a longitudinal beam matching to the wakefield requires either to design a bunch compressor or to find a bunch compressed solution through the RTM.

The transmission efficiency of the beam through the RTM has been improved up to $50 \%$, which was much better than the RTM with the thermionic gun. The expected beam charge is $500 \mathrm{pC} / \mathrm{bunch}$, that is enough for the LWFA experiments.
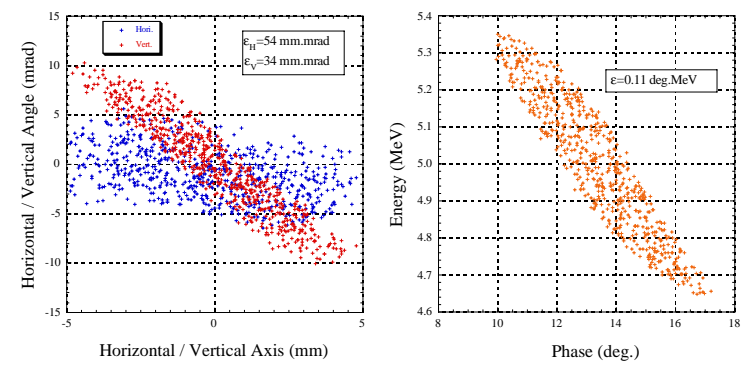

Figure 5: Acceptances of the RTM.

\subsection{Beam Extraction}

The RTM has two straight sections; one is non-dispersive where the accelerating tube is placed, and the other is dis-


Figure 6: Beam distributions at the exit of the RTM.

persive due to the $180^{\circ}$ bending magnet of the RTM. Because of the space limitation, beam extraction magnet is placed in the dispersive straight section. We designed a dispersion-compensated beam extraction section in order to avoid emittance growth caused by dispersion. The section was designed by employing transfer matrix technique to satisfy doubly achromatic conditions. Finally, we found that three bending magnets and four quadrupole magnets system can efficiently work with transverse focusing.

\section{SUMMARY}

We have designed the electron beam injection system oriented to effective electron acceleration by a laser wakefield. The RF photocathode and the race-track microtron are used for a low emittance beam generator and the expected transverse emittance satisfies the required conditions. The design work is still going on, and the left major work is to design short bunch generation and focusing sections.

\section{ACKNOWLEDGMENTS}

We are grateful to T. Ueda, K. Yoshii, T. Watanabe, and M. Uesaka of the University of Tokyo during the RF gun test.

\section{REFERENCES}

[1] K. Nakajima et al., Phys. Rev. Lett., 74 (1995) 4428; A. Modena et al., Nature (London) 377 (1995) 606; D. Umstadter et al., Science 273 (1996) 472; H. Dewa et al., Nucl. Instr. and Meth. in Phys. Res. A 410 (1998) 357; F. Amiranoff et al., Phys. Rev. Lett. 81 , (1998) 995.

[2] D. Umstadter et al., Phys. Rev. Lett., 76, (1996) 2073.

[3] M. Uesaka et al., Nucl. Instr. and Meth. in Phys. Res. A 410 (1998) 424.

[4] F. Sakai et al.,Proc. of 11th Symp. on Accel. Sci. and Tech. (1997) 473.

[5] D. T. Palmer et al., Proc. of Part. Accel. Conf. (1995) 982.

[6] M. Sugitani et al., Proc. of 1st Europ. Part. Accel. Conf. (1988) 596. 